\title{
4-Florobenzil Alkolün Konformasyon ve Orbital Etkileşimlerinin DFT Metodu ile Teorik Çalışması
}

\author{
Nihal KUŞ*1 (D), Saliha ILICAN² (D) \\ 1,2Eskişehir Teknik Üniversitesi, Fen Fakültesi, Fizik Bölümü, 26470, Eskişehir, Türkiye
}

(Alınış / Received: 26.03.2019, Kabul / Accepted: 07.11.2019, Online Yayınlanma / Published Online: 30.12.2019)

Anahtar Kelimeler

4-Florobenzil alkol,

DFT,

NBO,

Orbital etkileșimleri.
Özet: $\mathrm{Bu}$ çalışmada, 4-florobenzil alkol (4FBA) molekülünün konformasyonları DFT/B3LYP/6-311++G(d,p) metodu kullanılarak çalışılmıştır. Bileşiğin optimize edilmiş geometrisi ve enerjileri belirlenmiştir. 4FBA, minimum enerjili ve kararl yapıda iki konformasyona (4FBA-I ve 4FAB-II) sahiptir. İki konformasyon arasındaki bağıl elektronik enerji değeri 3,6 kJ mol-1 olarak hesaplanmıștır. Bariyer enerjisi 2,49 $\mathrm{kJ} \mathrm{mol}^{-1}$ gibi çok küçük bir değer olarak hesaplanmıştır. Bu durum, konformasyonel bir gevşemeye neden olarak, en kararlı duruma geçişi sağlayabilir. 4FBA-I ve 4FBA-II formlarının stabilizasyon enerjileri ve donör-akseptör etkileşimleri doğal bağ orbital (NBO) analizi ile yapılmıştır. NBO etkileşimlerinin yanında benzen halkasının karbon atomları üzerinden elektronik delokalizasyonun olduğu gözlenmiştir. $\mathrm{Bu}$ durum, $\pi \rightarrow \pi^{*}$ orbital etkileşimi ile açıklanabilir. Ayrıca, molekülün en yüksek dolu moleküler orbitali (HOMO) ve en düşük boș moleküler orbitali (LUMO) arasındaki enerji farkı hesaplanmıș, 4FBA-I ve 4FBA-II için sırasıyla 6,038 ve 6,142 eV olarak bulunmuştur.

\section{Theoretical Study of Conformational and Orbital Interactions of 4-Fluorobenzyl Alcohol by DFT Method}

\section{Keywords}

4-Fluorobenzyl alcohol, DFT,

NBO,

Orbital interactions.

\begin{abstract}
In this study, the conformations of 4-fluorobenzyl alcohol (4FBA) molecule were studied by using DFT/B3LYP/6-311++G(d,p) method. Optimized geometry and energies of compound were determined. 4FBA has two conformers (4FBA-I and 4FAB-II) with minimal energies and stable structures. The difference relative electronic energy of the two conformers were calculated about $3.6 \mathrm{~kJ} \mathrm{~mol}^{-1}$. Barrier energy was calculated as a very small value $\left(2,49 \mathrm{~kJ} \mathrm{~mol}^{-1}\right)$. This may cause conformational relaxation to the most stable conformer. The stabilization energies and donor-acceptor interactions of the 4FBA-I and 4FBA-II formations were performed by natural bond orbital (NBO) analysis. Besides to NBO interactions, electronic delocalization was observed over carbon atoms of the benzene ring, and this can be explained by $\pi \rightarrow \pi^{*}$ orbital interaction. In addition, the energy difference between the highest occupied molecular orbital (HOMO) and lowest unoccupied molecular orbital (LUMO) was calculated and found to be 6.038 and $6.142 \mathrm{eV}$ for 4FBA-I and 4FBA-II, respectively.
\end{abstract}

\section{Giriş}

Benzil alkol $\mathrm{C}_{7} \mathrm{H}_{8} \mathrm{O}$ formülüne sahip aromatik bir alkoldür. Vücutta, benzil alkol benzoik aside metabolize edilir. Koruyucu özelliği olduğu için yardımcı madde veya çözücü madde olarak kullanılır. Ayrica, anestezi özelliklerinin enjeksiyon bölgesindeki ağrıyı azalttığı için antibiyotikler, antienflamatuar veya nöroleptik ilaçlar gibi intramüsküler olarak uygulanan tıbbi ürünlerde de kullanılan yardımcı bir maddedir [1-3].
2002 yılında Avrupa Komisyonu SFC (Gida Bilimsel Komitesi), hayvan toksisitesi verilerine dayanarak, benzil alkol ile ilgili bazı araștırmalar yaparak yayınlamıştır [4]. Bird ve arkadaşları [5], gaz fazındaki 4-florobenzil alkol molekülünün önemli mikrodalga ve UV spektrumları sergilediğini açıklamışlar ve mikrodalga spektroskopisi ile yaptıkları deneyler sonucunda, benzen halkasının üst ve altta iki eșdeğer pozisyonda olduğunu ve ona ekli $\mathrm{C}_{2} \mathrm{OH}$ grubunun gölge şeklinde tutulduğunu rapor 
etmişlerdir. Guchait ve diğerleri [6] daha sonraki bir IR ve UV çalışmasında benzoik asit (BA) molekülünün teorik olarak konformasyon analizini yapmışlar ve yaptıkları hesaplarda da iki konformasyon bulmuşlardır. Mons ve çalışma arkadaşları [7], deneysel olarak IR ve UV çalışmaları sonucunda, BA molekülünün sadece gauche pozisyonunda olan bir konformasyonu olduğunu gözlemlemişlerdir. 2010 yılında Evangelisti ve çalışma arkadaşları [8], 2fluorobenzil alkol molekülünü dönme spektrumu ile incelemiş ve MP2/6-311++G(d,p) ile yaptıkları hesaplamalar sonucunda, $\mathrm{OC}_{\alpha}-\mathrm{C}_{1} \mathrm{C}_{2}$ ile karakterize edilen dihedral açısının yaklaşık 66 derece olmasıyla, gauche konformasyonunun en kararlı yapıda olduğunu yayınlamışlardır.

Formulü $\mathrm{C}_{7} \mathrm{H}_{7} \mathrm{FO}$ olan 4-florobenzil alkol (4FBA) molekülünün hidrojen bağ donör sayısı 1 , hidrojen bağ akseptör sayısı 2 ve döndürülebilir bağ sayısı 1 olup diğer bilinen adı (4-florofenil)metanoldür. 4FBA, benzil alkol yapısının bir homoloğudur ( $\mathrm{F}$ atomu yerine $\mathrm{H}$ atomunun gelmesi durumu) ve en basit bir aril alkoldür. Aril alkoller, organik sentezlerde yaygın kullanılan çözücülerdir. Formülleri oldukça basit olup, BA molekülünün halkaya bağlı $\mathrm{H}$ atomu ile $\mathrm{F}$ atomunun yer değiştirmesi ile oluşan bir türevidir.

$\mathrm{Bu}$ çalışmanın amacl, 4FBA molekülünün konformasyonlarını bulup, doğal bağ orbital (NBO) analizi sonuçlarından, konformasyonlara ait, orbital etkileşim analizlerini, atomik yüklerini ve en yüksek dolu moleküler orbitali (HOMO) ve en düşük boş moleküler orbitali (LUMO) arasındaki enerji aralığını teorik olarak incelemektir.

\section{Teorik Hesaplamalar}

4FBA molekülünün kuantum kimyasal hesaplamaları Gaussian 09 programı kullanılarak yapılmıştır [9]. Molekülün temel durumundaki denge geometrileri (bağ uzunlukları, bağ açıları ve dihedral açılar), yoğunluk fonksiyonel teorisinin (DFT) 6$311 /++G(d, p)$ düzeyinde ve B3LYP (Becke-3-LeeYang-Parr) temel seti kullanılarak optimize edilmiştir [10-12]. Bu yaklaşım, molekülün yapısı ve konformasyonlarının analizi için çok güçlü bir tekniktir. Elektronik (E) ve Gibbs serbest enerjisi (G) değerlerini belirlemek için, elde edilen verilere elektronik ve Gibbs serbest enerjisindeki düzeltmeler eklenmiş ve hesaplanmıștır. NBO analizi, molekül içindeki yük dağılımını daha iyi anlamak için Gaussian programında gerekli anahtar terimler kullanılarak yapılmıştır. Konformasyonların bağıl kararlı olma durumu, Weinhold ve çalışma grubunun geliştirdiği ve Gaussian 09 programında uygulanabilirliği olan NBO 3.1 programı kullanılarak analiz edilmiştir [13].

\section{Bulgular}

4FBA molekülünün optimizasyonu $311++G(d, p)$ yöntemi kullanılarak durumunda yapılmıştır. Hesaplanmış $\mathrm{C}_{1} \mathrm{C}_{12} \mathrm{O}_{13} \mathrm{H}$ dihedral açısının C-O bağı etrafında $30^{\circ}$ açı ile dönme işlemi sonucunda minumum enerjili iki durum bulunmuştur (Şekil 1).

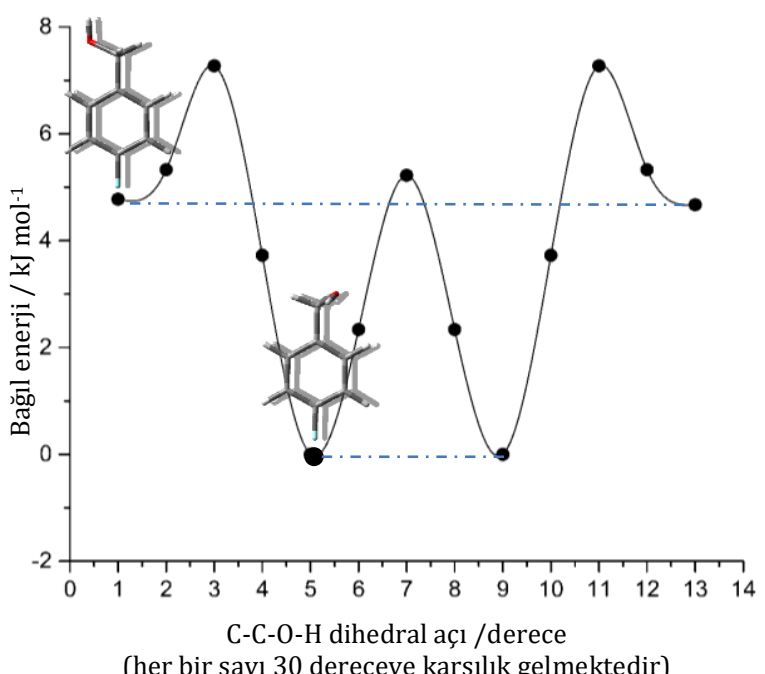

Şekil 1. DFT/B3LYP/6-311++G(d,p) metodu kullanılarak elde edilen, 4FBA molekülünün minimum durumu ve konformasyonunu gösteren potansiyel enerji grafiği.

Yapılan optimizasyon sonucunda, $\mathrm{C}_{1} \mathrm{C}_{12} \mathrm{O}_{13} \mathrm{H}$ dihedral açısının $\sim 5^{\circ}$ olma durumunda minimum, yani en kararlı yapı, ikinci minimum durumundaki yapı, $\mathrm{C}_{1} \mathrm{C}_{12} \mathrm{O}_{13} \mathrm{H}$ dihedral açısının $\sim 170^{\circ}$ olma durumunda gözlenmiştir. Bariyer enerjisinin 2,49 kJ mol-1 gibi çok küçük bir değere sahip olması, konformasyonel bir gevșeme ile en kararlı form olan 4FBA-I formuna dönmesine neden olabilir [14].

Sıfır nokta düzeltmiş enerjileri ile birlikte bağıl elektronik ve Gibbs enerjileri Tablo 1'de verilmiştir. Yapilan optimizasyon sonucundan, 4FBA-I'in 4FBA-II formundan 3,563 kJ mol-1 elektronik enerji değeri kadar daha kararlı yapıda olduğu ve her iki durumun C1 simetri grubuna ait olduğu bulunmuştur. Hesaplamalarda sıfır nokta düzeltmeleri dikkate alındığında, ikinci kararlı durumda olan 4FBA-II konformasyonu en kararlı yapı olan 4FBA-I formundan 3,190 kJ mol-1 enerji değeri kadar büyük bir enerjiye sahip olduğu belirlenmiștir.

Miller ve arkadaşları [15] BA molekülü için B3LYP/aug-cc-pVTZ yöntemi kullanarak yaptıkları hesaplamada, bağıl elektronik enerji değerini yaklaşık olarak $5 \mathrm{~kJ} \mathrm{~mol}^{-1}$ olarak hesaplamışlardır. BA molekülü için en kararlı yapıdaki bağıl enerji durumlarını Mons ve çalışma arkadaşları da [7] değerlendirmiş ve B3LYP/6-31+G(d) düzeyinde yaptıkları hesaplama sonucu ZPV ile birlikte bağıl enerji değerini 4,6 $\mathrm{kJ} \mathrm{mol}^{-1}, \mathrm{HF} / 6-31+\mathrm{G}(\mathrm{d}, \mathrm{p})$ hesaplarında $3,5 \mathrm{~kJ} \mathrm{~mol}^{-1}$ ve MP2/6-31+G(d,p) düzeyindeki hesaplamalarda $3,6 \mathrm{~kJ}$ mol$^{-1}$ olarak bulmuşlardır. $\mathrm{Bu}$ sonuçlar bize, kullanılan her yöntemin hesaplama sonuçlarının farklı olabileceğini ve moleküler optimizasyon için en iyi yöntemin hangisi olduğunu vermektedir. 
Tablo 1. Konformasyonların hesaplanmış simetrileri, bağıl elektronik enerjileri $(\Delta \mathrm{E})$, sıfır nokta düzeltilmiş enerjileri ile birlikte $(\Delta(E+Z P E))$ ve oda sıcaklığındaki bağıl Gibbs enerjileri $(\Delta G)$

\begin{tabular}{|c|c|c|c|c|c|c|}
\hline Konformasyon & $\begin{array}{c}\mathbf{E} \\
\text { Hartree }\end{array}$ & $\begin{array}{l}\text { E+ZPV } \\
\text { Hartree }\end{array}$ & $\begin{array}{c}\mathbf{G} \\
\text { Hartree }\end{array}$ & $\begin{array}{c}\Delta E \\
\text { kJ mol-1 }\end{array}$ & 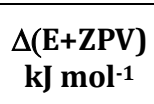 & $\begin{array}{c}\Delta G \\
\text { kJ mol-1 }\end{array}$ \\
\hline 4FBA-I & $-446,020$ & $-446,054$ & $-446,145$ & 0 & 0 & 0 \\
\hline 4FBA-II & $-446,019$ & $-446,052$ & $-446,143$ & 3,563 & 3,190 & 4,476 \\
\hline
\end{tabular}

Oda sıcaklığında (298 K), gaz fazında, DFT(B3LYP)/6$311++G(d, p)$ metodu kullanılarak yapılan hesaplarda bağll Gibbs enerjisi 4FBA-I formunda 4,476 kJ mol-1 enerji değeri kadar daha yüksek kararlığa sahip olduğu görülmüştür (Tablo 1). Yapılan hesaplamada 4FBA-I durumundaki popülasyonun, 4FBA-II durumundan daha fazla olduğu belirlenmiștir. 4FBA molekülü ve konformasyonunun elde edilen minimum durumları Şekil 2'de verilmiştir.
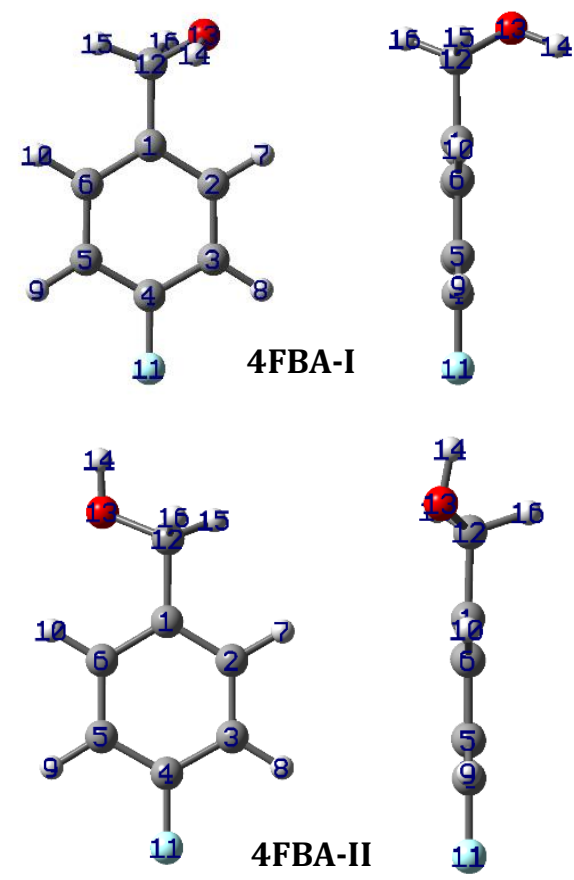

Şekil 2. 4FBA molekülünün numaralandırılmış haliyle, DFT(B3LYP)/6-311++G(d,p) kullanılarak yapilan hesaplamada elde edilen minimum durumları (İki farklı açıdan gösterilmiştir).

4FBA molekülünün yapısı $\tau_{1}(\mathrm{CCCO})$ ve $\tau_{2}(\mathrm{CCOH})$ olmak üzere iki dihedral açıya göre tanımlanabilir. DFT-B3LYP/6-311++G(d,p) yaklaşımı ile yapılan hesaplamalarda her iki dihedral açısı dikkate alınmış olup, iki farklı minimum gözlenmiștir. Evangelisti ve arkadaşları [8], oda sıcaklığında, dönme spektrumları ile yaptıkları analizde 2FBA molekülünün iki minimumda karalı forma sahip olduğunu bulmuşlar ve gauche olarak isimlendirdikleri (şimdiki çalışmada, 4FBA-I durumuna karşılık gelen) yapıyı en kararlı yapı olarak rapor etmişlerdir. MP2/6$311++G(d, p)$ düzeyinde yaptıkları hesaplamalar sonucunda, elektronik bağll enerjiyi 2,87 kJ mol-1 olarak bulmuşlar. Şimdiki çalışmada, DFT(B3LYP)/6$311++G(d, p)$ temel setinde yapilan hesaplamada,
3,6 kJ mol-1 olarak hesaplanmıştır. Bu fark, hem kullanılan yöntemin farklı olmasından, hem de F atomunun 2. yerine 4. karbon atomundan bağlanmasından kaynaklandığı düşünülebilir.

Alves ve çalışma arkadaşları [16] da 3FBA ve 4FBA molekülleri için potansiyel enerji yüzeyini, DF-LMP2F12/DF-LMP2/cc-pVQZ teori seviyesinde hesaplamışlar ve sonuçlarda birbirine çok yakın iki minimum gözlemişlerdir. Crowder [17] 1979 yılında $o$-florobenzil alkol molekülünün titreșimlerini Cs2 ve $\mathrm{CCl}_{4}$ çözücülerinde IR spektroskopisi ile incelemiştir.

Benzer yapıdaki her iki konformasyonun yapısal bağıl kararlılığını, hibridizasyonunu ve Van der Waals etkileşimlerini ayrıntılı olarak incelemek için NBO yöntemi kullanılmıştır. $\mathrm{Bu}$ yöntem kullanılarak, her iki forma ait stabilizasyon enerjileri, orbital etkileşimleri, atomik yükler ve HOMO-LUMO enerjileri araştırılmıştır. Orbital etkileşimlerde bulunan, $\pi$ ve $\pi^{*}$ sembolleri, Lewis yapısının dolu ve boş yörüngelerini, LP sembolü ise bağ yapmamış değerlik elektron çift orbitalini göstermektedir. Boş orbital donör, dolu orbital akseptör olarak tanımlanır.

DFT(B3LYP)/6-311++G(d,p) temel setinde yapilan hesaplamalarda, Schrödinger dalga fonksiyonlarının sonucunda, Fock matrisinin ikinci dereceden pertürbasyon teori analizi ile molekül orbital etkileşimleri belirlenmiştir. Stabilizasyon enerjileri aşağıda verilen Fock formülü kullanılarak hesaplanabilir.

$$
E(2)=\Delta E_{i j}=q_{i} \frac{F_{i j}^{2}}{\varepsilon_{j}-\varepsilon_{i}}
$$

Burada; $F_{i j}^{2}, i$ ve $j$ NBO orbitalleri arasındaki Fock matris elemanı, $\varepsilon_{j}$ ve $\varepsilon_{i}$ akseptör ve donör NBO orbitalleri, $q_{i}$ ise donör orbitalinin doluluk oranıdır [18].

Tablo 2'de stabilizasyon enerjisi kJ mol-1 olarak verilen 4FBA molekülünün konformasyonlarına ait NBO analizi sonuçlarından bulunan orbital çiftleri verilmiştir.

Bunun yanında ayrıca, bağ dipollerini içeren ve stabilizasyon enerjisinin \%10 değerinden büyük olan (benzen halkasına ait) orbital çiftleri seçilerek, molekül içi etkileşimleri içeren orbital şemaları çizilmiştir (Şekil 3). 
Tablo 2. 4FBA-I ve 4FBA-II konformasyonları için NBO bazında, Fock matrisinin B3LYP/6-311++G(d,p) hesaplarından elde edilen ikinci mertebeden pertürbasyon teorisi analizi ile seçilen NBO çiftleri için stabilizasyon enerjileri (\%10 değerinden büyük olan stabilizasyon enerji değerleri dikkate alınmıștır. LP, bağ yapmamıș değerlik elektron çift orbitalidir. Şekil 1'deki atom numaralandırmasına bakınız).

\begin{tabular}{|c|c|c|c|c|c|c|c|c|}
\hline \multirow[b]{2}{*}{ Orbital çifti } & \multirow{2}{*}{$\begin{array}{c}\text { Donör } \\
\text { NBO }\end{array}$} & \multirow{2}{*}{$\begin{array}{c}\text { Akseptör } \\
\text { NBO }\end{array}$} & \multicolumn{3}{|c|}{ 4FBA-I } & \multicolumn{3}{|c|}{ 4FBA-II } \\
\hline & & & $\begin{array}{c}E(2) \\
(\mathrm{kJ})\end{array}$ & $\begin{array}{l}\varepsilon^{-} \varepsilon_{i} \\
\mathbf{a u}\end{array}$ & $\begin{array}{l}F_{i j} \\
\text { au }\end{array}$ & $\begin{array}{c}E(2) \\
(\mathrm{kJ} \mathrm{mol}-1)\end{array}$ & $\begin{array}{l}\varepsilon_{j}{ }^{-} \varepsilon_{i} \\
\mathrm{au}\end{array}$ & $\begin{array}{l}F_{i j} \\
\text { au }\end{array}$ \\
\hline $\mathbf{A}$ & $\pi(\mathrm{C} 1-\mathrm{C} 6)$ & $\pi *(\mathrm{C} 2-\mathrm{C} 3)$ & 88,95 & 0,28 & 0,069 & 93,97 & 0,27 & 0,070 \\
\hline B & $\pi(\mathrm{C} 1-\mathrm{C} 6)$ & $\pi^{*}(\mathrm{C} 4-\mathrm{C} 5)$ & 80,42 & 0,27 & 0,065 & 84,39 & 0,27 & 0,066 \\
\hline C & $\pi(\mathrm{C} 2-\mathrm{C} 3)$ & $\pi^{*}(\mathrm{C} 1-\mathrm{C} 6)$ & 78,58 & 0,29 & 0,066 & 78,54 & 0,29 & 0,067 \\
\hline D & $\pi(\mathrm{C} 2-\mathrm{C} 3)$ & $\pi^{*}(\mathrm{C} 4-\mathrm{C} 5)$ & 96,01 & 0,28 & 0,072 & 92,46 & 0,28 & 0,071 \\
\hline $\mathbf{E}$ & $\pi(\mathrm{C} 4-\mathrm{C} 5)$ & $\pi^{*}(\mathrm{C} 1-\mathrm{C} 6)$ & 85,02 & 0,30 & 0,070 & 84,48 & 0,30 & 0,070 \\
\hline $\mathbf{F}$ & $\pi(\mathrm{C} 4-\mathrm{C} 5)$ & $\pi *(\mathrm{C} 2-\mathrm{C} 3)$ & 76,08 & 0,30 & 0,066 & 80,63 & 0,29 & 0,067 \\
\hline G & LP(F11) & $\pi^{*}(\mathrm{C} 4-\mathrm{C} 5)$ & 75,37 & 0,43 & 0,085 & 73,65 & 0,43 & 0,085 \\
\hline
\end{tabular}
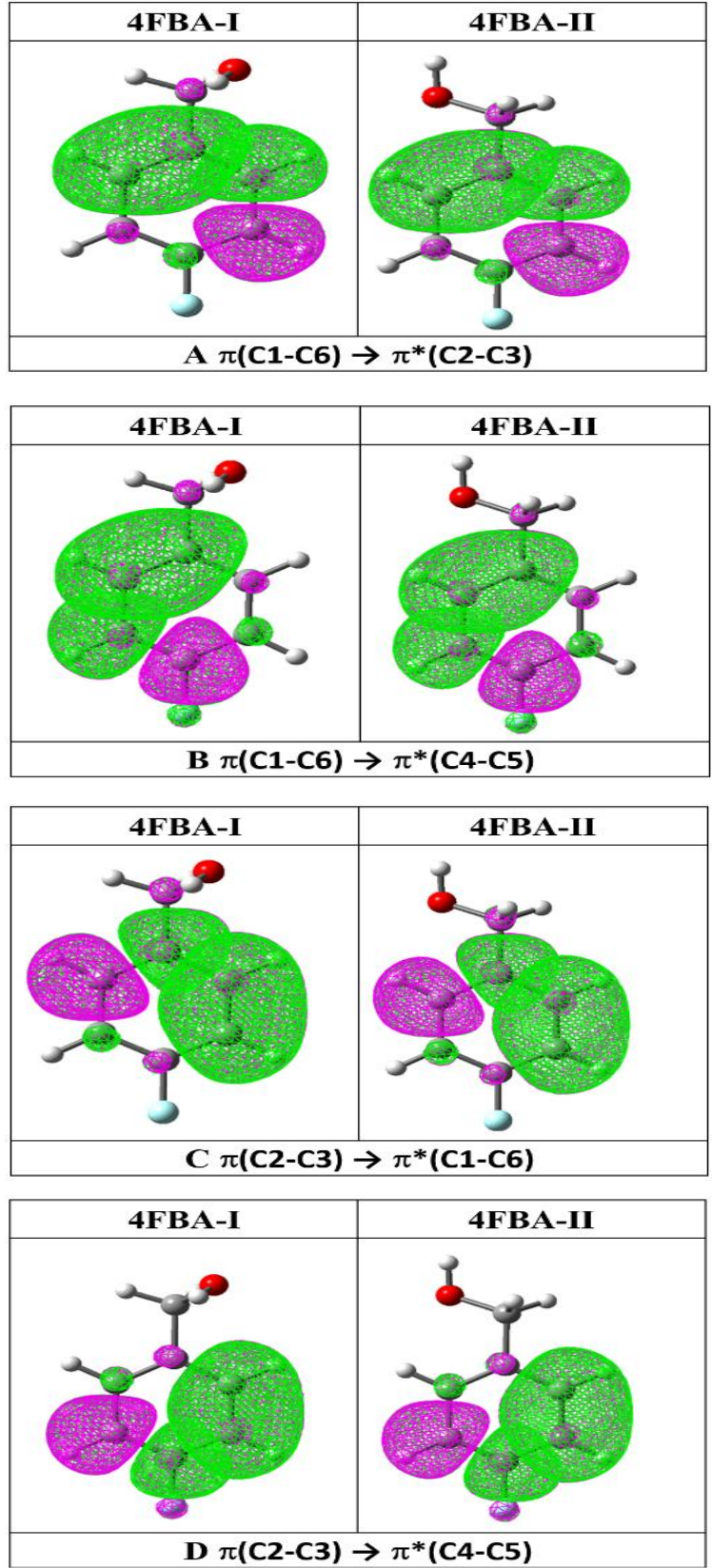
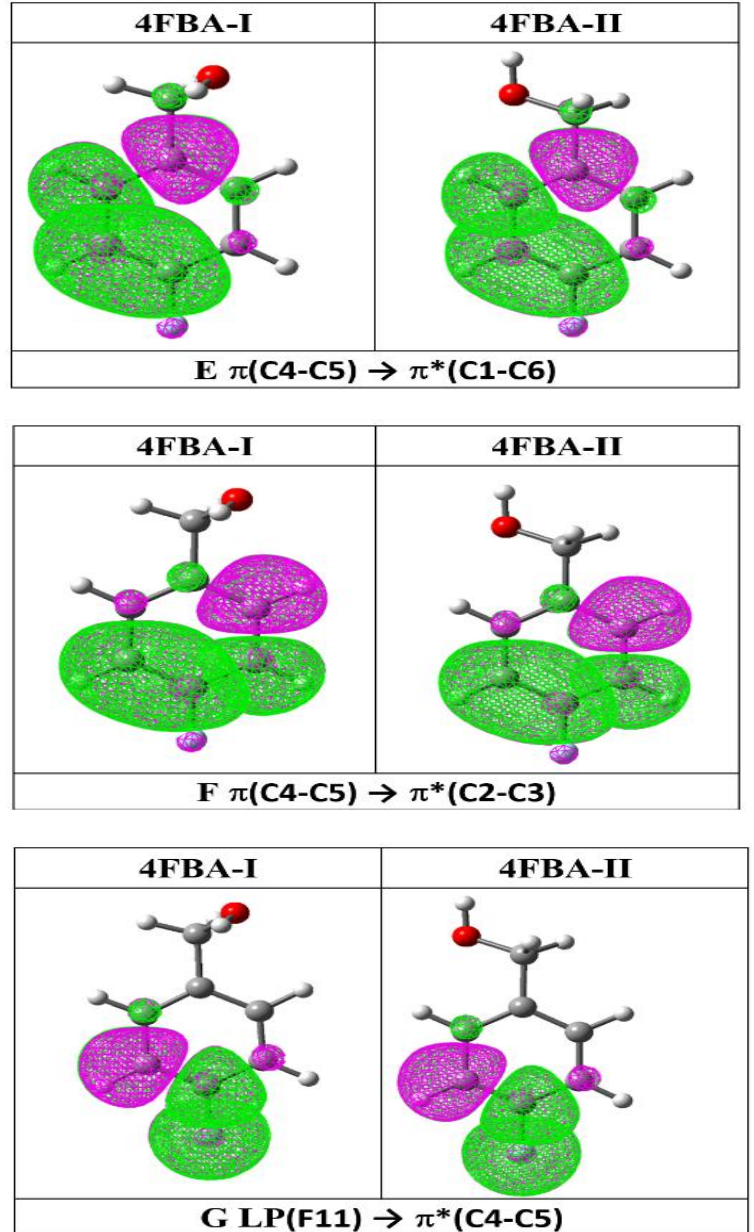

Şekil 3. Baskın orbital etkileşimleri gösteren B3LYP/6-311++G(d,p) teorisi düzeyinde hesaplanan 4FBA-I ve 4FBA-II konformasyonları için seçilen NBO'ların elektron yoğunluğu yüzeyleri (bkz. Tablo 2). Elektron yoğunluklarının izodeğerleri, 0,02 e'ye eşittir. Yeşil ve pembe renkler, negatif ve pozitif dalga fonksiyon işaretlerine karşılık gelmektedir. Atomların renk kodları: kırmızı, O; gri, C; beyaz, H; açık mavi, F 
Çeşitli "orbital-orbital" ve "bağ yapmamış değerlik elektron çifti-orbital" çakışması nedeniyle çeşitli molekül içi etkileșimler oluşmuştur. Sonuçlardan, NBO etkileşimlerinin yanında benzen halkasının karbon atomları üzerinden elektronik delokalizasyonun olduğu gözlenmiş ve bu durum $\pi \rightarrow \pi^{*}$ orbital etkileșimi ile açıklanmıştır.

Molekül içi yük transferinden dolayı ve donör akseptör üzerindeki $\pi$-elektron bulutunun hareketinden dolayı, molekül daha fazla polarize hale gelir. Ayrıca F atomuna bağlı olarak her iki konformasyona ait, güçlü $L P \rightarrow \pi^{*}$ donör-akseptör etkileşimi ( $\mathrm{F}$ atomu ile C4-C5 anti bağ etkileşimi) görülmüştür. Tablo 3 ve 4 'te verilen tüm etkileşimler p-hibritleşmesinden kaynaklanmaktadır. Stabilizasyon enerjileri \%10 değerinden büyük olan dominant orbital etkileşimler dikkate alındığında, hesaplanan sonuçlara göre, orbital etkileşimleri 4FBA-I durumunda, $\sim 8 \mathrm{~kJ} \mathrm{~mol}^{-1}$ enerji değeri kadar 4FBA-II formundan daha güçlüdür. Buna göre; $\pi(\mathrm{C} 2-$ $\mathrm{C} 3) \rightarrow \pi^{*}(\mathrm{C} 1-\mathrm{C} 6), \quad \pi(\mathrm{C} 2-\mathrm{C} 3) \rightarrow \pi^{*}(\mathrm{C} 4-\mathrm{C} 5), \quad \pi(\mathrm{C} 4-$
$\mathrm{C} 5) \rightarrow \pi^{*}(\mathrm{C} 1-\mathrm{C} 6), \quad \pi(\mathrm{C} 4-\mathrm{C} 5) \rightarrow \pi^{*}(\mathrm{C} 2-\mathrm{C} 3) \quad$ ve $\mathrm{LP}(\mathrm{F} 11) \rightarrow \pi^{*}(\mathrm{C} 4-\mathrm{C} 5)$ orbital etkileşimleri 4FBA-I formunda sirasiyla 0,$04 ; 3,55 ; 0,54$ ve $1,71 \mathrm{kJmol}^{-1}$ enerji değeri kadar 4FBA-II formuna göre daha güçlüdür. $\pi(\mathrm{C} 1-\mathrm{C} 6) \rightarrow \pi^{*}(\mathrm{C} 2-\mathrm{C} 3), \quad \pi(\mathrm{C} 1-\mathrm{C} 6) \rightarrow \pi^{*}(\mathrm{C} 4-$ $\mathrm{C} 5)$ ve $\pi(\mathrm{C} 4-\mathrm{C} 5) \rightarrow \pi^{*}(\mathrm{C} 2-\mathrm{C} 3)$ orbital etkileşimleri 4FBA-II formunda sirasiyla 5,02; 3,97ve 4,56 kJmol-1 enerji değeri kadar 4FBA-I formuna göre daha güçlüdür. Şekil 3'ten de görüldüğü gibi, konjüge $\pi$ sisteminde elektron yoğunluğu tüm $\mathrm{C}$ atomları üzerinde delokalize haldedir. Benzen halkasına bağlı F atomu da, elektron yoğunluğunun az olduğu durumu açıklamaktadır.

4FBA-I ve 4FBA-II formları için seçilmiş NBO hibritleri ile Lewis yörüngeleri (işgal edilmiş bağ veya yalnız çift) Tablo 3 ve Tablo 4'te listelenmiștir.

NBO orbitallerinin valans hibrit analizi sonucunda, benzen halkasına ait $\mathrm{C}$ atomları birbirleri ile polarize olurken $\mathrm{F}$ atomu C-C ile güçlü polarizasyona neden olmuştur.

Tablo 3. B3LYP/6-311++G(d,p) yöntemi ile hesaplanmış, 4FBA-I formuna ait stabilizasyon enerjilerinin en yüksek olduğu durumlara ait doluluk oranları, \% katsaylları ve hibritleșme durumları

\begin{tabular}{|c|c|c|c|c|c|}
\hline \multirow{2}{*}{ Grup } & \multirow{2}{*}{ NBO } & \multirow{2}{*}{ Doluluk oran } & \multicolumn{2}{|c|}{ (\%) Katsayılar a } & \multirow{2}{*}{ Hibritleşme $^{\mathbf{b}}$} \\
\cline { 3 - 5 } & & $\boldsymbol{A}$ & $\boldsymbol{B}$ & \\
\hline donör & $\pi($ C1-C6) & 1,66387 & 51,15 & 48,85 & $0,7152 \mathrm{p}+0,6989 \mathrm{p}$ \\
\hline akseptör & $\pi^{*}(\mathrm{C} 2-\mathrm{C} 3)$ & 0,32097 & 51,98 & 48,02 & $0,7210 \mathrm{p}-0,6930 \mathrm{p}$ \\
\hline donör & $\pi(\mathrm{C} 2-\mathrm{C} 3)$ & 1,68374 & 48,02 & 51,98 & $0,6930 \mathrm{p}+0,7210 \mathrm{p}$ \\
\hline akseptör & $\pi *$ (C4-C5) & 0,36417 & 51,45 & 48,55 & $0,7173 \mathrm{p}-0,6968 \mathrm{p}$ \\
\hline donör & $\pi($ C4-C5) & 1,66591 & 48,55 & 51,45 & $0,6968 \mathrm{p}+0,7173 \mathrm{p}$ \\
\hline akseptör & $\pi *($ C1-C6) & 0,351129 & 48,85 & 51,15 & $0,6989 \mathrm{p}-0,7152 \mathrm{p}$ \\
\hline donör & LP(3)F11 & 1,92773 & & & $\mathrm{p}$ \\
\hline
\end{tabular}

a $A$ ve $B$ değerleri, iki atomun atomik orbitallerinin NBO orbitalleri için bir bağ oluşturarak, bu çifleri oluşturan atomların katkılarına karşılık gelir ve polarizasyonların katsayılarından elde edilir.

b Hibrit orbitallerinin tanımı.

Tablo 4. B3LYP/6-311++G(d,p) yöntemi ile hesaplanmıș, 4FBA-II formuna ait stabilizasyon enerjilerinin en yüksek olduğu durumlara ait doluluk oranları, \% katsayıları ve hibritleşme durumları

\begin{tabular}{|c|c|c|c|c|c|}
\hline \multirow{2}{*}{ Grup } & \multirow{2}{*}{ NBO } & \multirow{2}{*}{ Doluluk oranı } & \multicolumn{2}{|c|}{ (\%) Katsayılar a } & \multirow{2}{*}{ Hibritleşme b } \\
\hline & & & $A$ & $B$ & \\
\hline donör & $\pi(\mathrm{C} 1-\mathrm{C} 6)$ & 1,64877 & 51,41 & 48,59 & $0,7170 p+0,6971 p$ \\
\hline akseptör & $\pi^{*}(\mathrm{C} 2-\mathrm{C} 3)$ & 0,34667 & 51,22 & 48,78 & $0,7157 p-0,6984 p$ \\
\hline donör & $\pi(\mathrm{C} 2-\mathrm{C} 3)$ & 1,69031 & 48,78 & 51,22 & $0,6984 p+0,7157 p$ \\
\hline akseptör & $\pi *(\mathrm{C} 4-\mathrm{C} 5)$ & 0,37114 & 51,09 & 48,91 & $0,7148 p-0,6993 p$ \\
\hline donör & $\pi(\mathrm{C} 4-\mathrm{C} 5)$ & 1,66254 & 48,91 & 51,09 & $0,6993 p+0,7148 p$ \\
\hline akseptör & $\pi *(\mathrm{C} 1-\mathrm{C} 6)$ & 0,34651 & 48,59 & 51,41 & $0,6971 p-0,7170 p$ \\
\hline donör & LP(3)F11 & 1,92961 & & & $\mathrm{p}$ \\
\hline
\end{tabular}

a $A$ ve $B$ değerleri, iki atomun atomik orbitallerinin NBO orbitalleri için bir bağ oluşturarak, bu çifleri oluşturan atomların katkılarına karşıllı gelir ve polarizasyonların katsayılarından elde edilir.

b Hibrit orbitallerinin tanımı 
NBO hesaplarının sonuçlarından, 4FBA-I ve 4FBA-II durumlarına ait doğal atomik yükler ve Mulliken yükler elde edilmiştir. Moleküllere ait yük tabloları Tablo 5 ve 6'da verilmiştir. 4FBA-I formunun benzen halkası için atomik yüklerin en yüksek pozitif olanı C4 atomuna ait olup, 0,41146e değerindedir. Benzen halkasina bağlı $\mathrm{F}$ atomu ise $-0,35458 \mathrm{e}$ değerinde negatif yük değerindedir. Bu durumda C-F bağının polarize durumunun yüksek olduğu söylenebilir. Aynı durum 4FBA-II durumu için de geçerlidir. Atomik yüklere bağlı olarak en yüksek polarizasyonun 0 atomu ile $\mathrm{H}$ atomu arasında olduğu Tablo 3 ve 4 'ten görülmektedir.

(4FBA-I için; $\mathrm{H}_{14}=0,45394$ e ve $\mathrm{O}_{13}=-0,72584 \mathrm{e}$; 4FBA-II için; $\mathrm{H}_{14}=0,46075$ e ve $\mathrm{O}_{13}=-0,73456 \mathrm{e}$ )

Tablo 5. 4FBA-I için B3LYP/6-311++G(d,p) hesaplarından elde edilen Mulliken ve doğal atomik yükler (e biriminde, e $=1.6 \times 10^{-19} \mathrm{C}$; Atomların numaralandırılmış durumu Şekil 2'de verilmiștir)

\begin{tabular}{|c|c|c|c|}
\hline \multicolumn{5}{|c|}{ 4FBA-I } \\
\hline Atom & No & NBO & Mulliken Yük \\
\hline C & 1 & $-0,09146$ & 1,113286 \\
\hline C & 2 & $-0,17146$ & $-0,490961$ \\
\hline C & 3 & $-0,25953$ & 0,399033 \\
\hline C & 4 & 0,41146 & $-0,747785$ \\
\hline C & 5 & $-0,26360$ & 0,445670 \\
\hline C & 6 & $-0,18159$ & $-1,248431$ \\
\hline H & 7 & 0,21784 & 0,176329 \\
\hline H & 8 & 0,22332 & 0,201688 \\
\hline H & 9 & 0,22316 & 0,204563 \\
\hline H & 10 & 0,20578 & 0,135218 \\
\hline F & 11 & $-0,35458$ & $-0,172293$ \\
\hline C & 12 & $-0,03392$ & $-0,368121$ \\
\hline O & 13 & $-0,72584$ & $-0,233198$ \\
\hline H & 14 & 0,45394 & 0,228888 \\
\hline H & 15 & 0,15975 & 0,177987 \\
\hline H & 16 & 0,18671 & 0,178126 \\
\hline
\end{tabular}

Tablo 6. 4FBA-II için B3LYP/6-311++G(d,p) hesaplarından elde edilen Mulliken ve doğal atomik yükler (e biriminde, e $=1.6 \times 10^{-19} \mathrm{C}$; Atomların numaralandırılmıș durumu Şekil 3.2'de verilmiștir)

\begin{tabular}{|c|c|c|c|}
\hline \multicolumn{5}{|c|}{ 4FBA-II } \\
\hline Atom & No & NBO & Mulliken \\
\hline C & 1 & $-0,07129$ & 0,981455 \\
\hline C & 2 & $-0,18469$ & $-1,057312$ \\
\hline C & 3 & $-0,26130$ & 0,459517 \\
\hline C & 4 & 0,40597 & $-0,771567$ \\
\hline C & 5 & $-0,25811$ & 0,441223 \\
\hline C & 6 & $-0,17799$ & $-0,611061$ \\
\hline H & 7 & 0,20333 & 0,146075 \\
\hline H & 8 & 0,22148 & 0,195601 \\
\hline H & 9 & 0,22147 & 0,199074 \\
\hline H & 10 & 0,22716 & 0,196628 \\
\hline F & 11 & $-0,35746$ & $-0,176799$ \\
\hline C & 12 & $-0,02644$ & $-0,309118$ \\
\hline O & 13 & $-0,73456$ & $-0,281728$ \\
\hline H & 14 & 0,46075 & 0,248226 \\
\hline H & 15 & 0,16393 & 0,172841 \\
\hline H & 16 & 0,16776 & 0,166945 \\
\hline
\end{tabular}
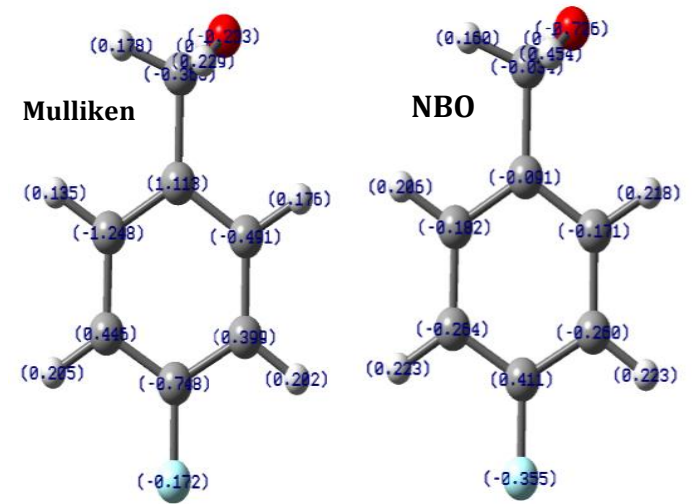

Şekil 4. 4FBA-I için B3LYP/6-311++G(d,p) hesaplarından elde edilen Mulliken ve doğal atomik yükler (e biriminde, e $=1.6 \times 10^{-19} \mathrm{C}$; Atomların numaralandırılmıș durumu Şekil 2 'de verilmiştir)
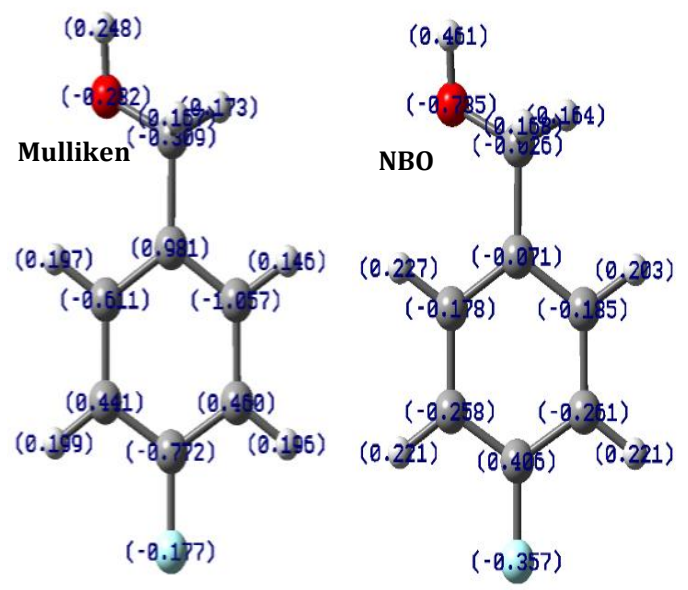

Şekil 5. 4FBA-II için B3LYP/6-311++G(d,p) hesaplarından elde edilen Mulliken ve doğal atomik yükler (e biriminde, e $=1.6 \times 10^{-19} \mathrm{C}$; Atomların numaralandırılmış durumu Şekil 2'de verilmiștir)

Mulliken atomik yüklerden görüldüğü gibi, $\mathrm{O}$ atomunun bağlanma pozisyonuna göre, $\mathrm{C}_{2}$ ve $\mathrm{C}_{6}$ atomlarının yük değerleri, diğer atomların yüklerine göre daha fazla değişmiştir.

(4FBA-I için; $\mathrm{C}_{2}=-0,490961$ e ve $\mathrm{C}_{6}=-1,248431 \mathrm{e}$; 4FBA-II için; $\mathrm{C}_{2}=-1,057312$ e ve $\mathrm{C}_{6}=-0,611061 \mathrm{e}$ )

HOMO ve LUMO enerjileri, molekülde yük transferinin gerçekleșip gerçekleşmediğini göstermektedir. 4FBA-I ve 4FBA-II durumlarına ait B3LYP/6-311++G(d,p) yöntemi ile hesaplanmış HOMO-LUMO enerji farkı sirasıly 6,038 ve $6,142 \mathrm{eV}$ olarak bulunmuștur. HOMO ve LUMO orbitallerinin negatif ve pozitif yoğunluk dağılımları Şekil 6'da verilmiştir.

\section{Tartışma ve Sonuç}

4FBA molekülünün kararlı durumundaki geometrisi ve kuantum kimyasal analizleri DFT-B3LYP/6$311++G(d, p)$ metodu kullanılarak gaz fazında yapılmıştır. 


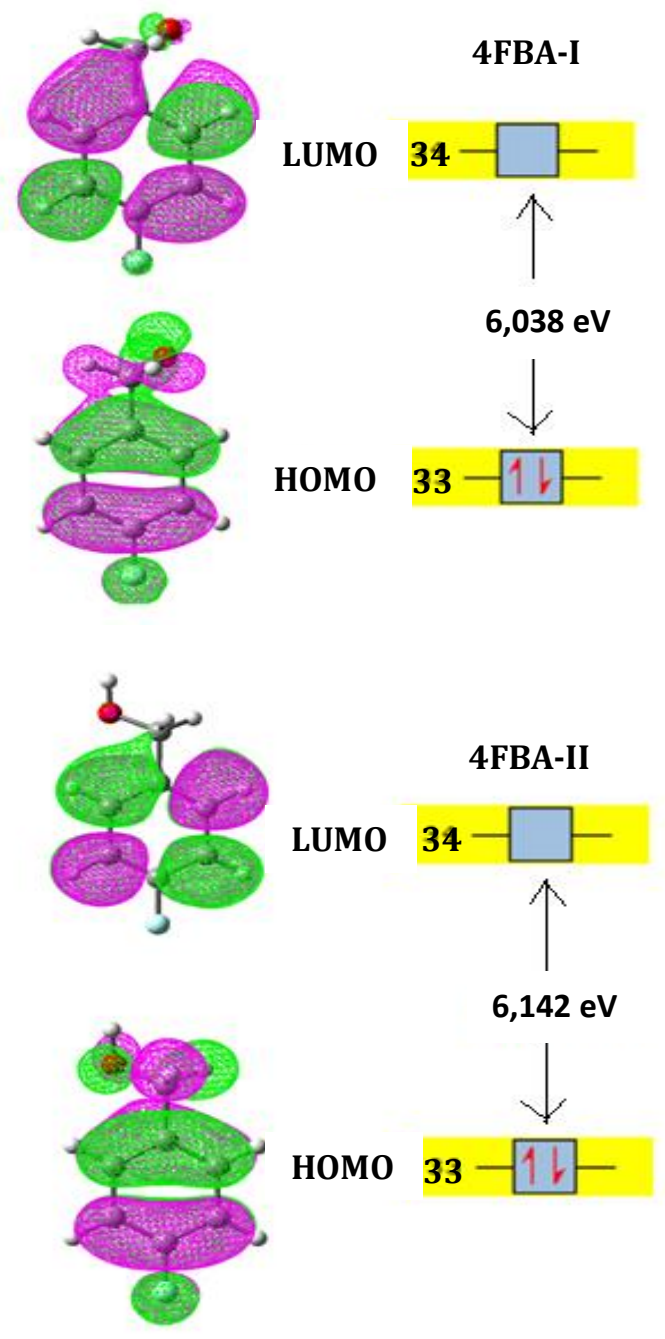

Şekil 6. FBA-I ve 4FBA-II formları için atomik orbital bileşimlerini gösteren HOMO-LUMO enerji aralıkları

Kararlı durumda iki minimum enerjiye sahip olduğu bulunmuş ve elektronik enerji farkı iki durum arasında 3,19 $\mathrm{kJ} \mathrm{mol}^{-1}$ olarak hesaplanmıștır (sıfır nokta enerjisi ile birlikte). 4FBA-I ve 4FBA-II minimum kararlı durumlarına ait bağıl stabilizasyonlar NBO yöntemi ve Fock matris denklemi kullanılarak açıklanmış ve NBO orbitallerine ait en yüksek stabilizasyon enerjilerinin hibritleşme durumları ve miktarları yüzde olarak hesaplanmıștır. NBO analizi sonucunda 4FBA-I formu için $\pi(\mathrm{C} 2-\mathrm{C} 3) \rightarrow \pi^{*}(\mathrm{C} 4-\mathrm{C} 5)$ stabilitesinin en yüksek enerjiye sahip olduğu $\left(96,01 \mathrm{~kJ} \mathrm{~mol}^{-1}\right)$, 4FBA-II formu için ise $\pi(\mathrm{C} 1-\mathrm{C} 6) \rightarrow \pi^{*}(\mathrm{C} 2-\mathrm{C} 3)$ olduğu görülmüştür (93,97 kJ mol-1). HOMO-LUMO enerji farkı hesaplanarak, orbital dağılımları çizilmiştir. 4FBA_II formunun HOMO-LUMO enerjisi 4FBA-I formundan 0,104 eV değeri kadar daha fazla olduğu görülmüştür.

\section{Kaynakça}

[1] EPA; health and environmental effects document for benzyl alcohol; September 1989.
[2] Frederick, H., Lovejoy, Jr. M. D. 1982. Fatal benzyl alcohol poisoning in a neonatal intensive care unit. Am. J. Dis. Child., 136(11), 974-975.

[3] Gershanik, J., Boecler, B., Ensley, H., McCloskey, S., George, W. 1982. The gasping syndrome and benzyl alcohol poisoning. N. Engl. J. Med., 307(22),1384-1388.

[4] Opinion of the Scientific Committee on Food on Benzyl alcohol, 2002. (Erişim Tarihi: 18.03.2019).http://ec.europa.eu/food/fs/sc/scf /out138_en.pdf.

[5] Bird, R. G., Nikolaev, A. E., Prat, D. W. 2011. Microwave and UV Excitation Spectra of 4Fluorobenzyl Alcohol at High Resolution. S0 and S1 Structures and Tunneling Motions along the Low Frequency $-\mathrm{CH}_{2} \mathrm{OH}$ Torsional Coordinate in Both Electronic States. J. Phys. Chem. A, 115, 11369-11377.

[6] Guchhait, N., Ebata, T., Mikami, N. 1999. Discrimination of Rotamers of Aryl Alcohol Homologues by Infrared-Ultraviolet DoubleResonance Spectroscopy in a Supersonic Jet. J. Am. Chem. Soc., 121, 5705-5711.

[7] Mons, M., Robertson, E. G., Simons, J. P. 2000. Intra- and Intermolecular $\pi$-Type Hydrogen Bonding in Aryl Alcohols: UV and IR-UV Ion Dip Spectroscopy. J. Phys. Chem. A, 104 (7), 14301437.

[8] Evangelisti, L., Favero, L. B., Caminati, W. 2010. Rotational spectrum of 2-fluorobenzyl alcohol. J. Mol. Struc., 978, 279-281.

[9] Frisch, M. J., Trucks, G. W., Schlegel, H. B., Scuseria, G. E., Robb, M. A., Cheeseman, J. R., Scalmani, G., Barone, V., Mennucci, B., Petersson, G. A., Nakatsuji, H., Caricato, M., Li, X., Hratchian, H. P., Izmaylov, A. F., Bloino, J., Zheng, G., Sonnenberg, J. L., Hada, M., Ehara, M., Toyota, K., Fukuda, R., Hasegawa, J., Ishida, M., Nakajima, T., Honda, Y., Kitao, O., Nakai, H., Vreven, T., Montgomery, J. A., Peralta Jr, J. E., Ogliaro, F., Bearpark, M., Heyd, J. J., Brothers, E., Kudin, K. N., Staroverov, V. N., Kobayashi, R., Normand, J., Raghavachari, K., Rendell, A., Burant, J. C., Iyengar, S. S., Tomasi, J., Cossi, M., Rega, N., Millam, J. M., Klene, M., Knox, J. E., Cross, J. B., Bakken, V., Adamo, C., Jaramillo, J., Gomperts, R., Stratmann, R. E., Yazyev, O., Austin, A. J., Cammi, R., Pomelli, C., Ochterski, J. W., Martin, R. L., Morokuma, K., Zakrzewski, V. G., Voth, G. A., Salvador, P., Dannenberg, J. J., Dapprich, S., Daniels, A. D., Farkas, O., Foresman, J. B., Ortiz, J. V., Cioslowski, J., Fox, D. J., 2009, Gaussian 09, Revision A.0.2, Gaussian, Inc., Wallingford CT.

[10] Becke, A. D. 1988. Density-functional exchangeenergy approximation with correct asymptotic behavior. Phys. Rev. A, 38, 3098-3100. 
[11] Clark, T., Chandrasekhar, J., Spitznagel, G. W., Schleyer, P. V. R. 1983. Efficient diffuse functionaugmented basis sets for anion calculations. III. The 3-21+G basis set for first-row elements, Li-F. J. Comput. Chem., 4, 294-301.

[12] Lee, C. T., Yang, W. T., Parr, R. G. 1988. Development of the Colic-Salvetti correlationenergy formula into a functional of the electron density. Phys. Rev. B, 37, 785-789.

[13] Reed, A. E., Curtiss, L. A., Weinhold, F. 1988. Intermolecular interactions from a natural bond orbital, donor-acceptor viewpoint. Chem. Rev., $88,899-926$.

[14] Ruoff, R. S., Klots, T. D., Emilson, T., Gutowski, H. S. Relaxation of conformers and isomers in seeded supersonic jets of inert gases. J. Chem. Phys., 93, 3142-3150.

[15] Miller, B. J., Kjaergaard, H. G., Hattori, K., Ishiuchi,
S., Fujii, M. 2008. The most stable conformer of benzyl alcohol. Chemical Physics Letters, 466, 21-26.

[16] Alves, T.V., S.-Carballido, L., Ornellas, F.R., F.Ramos, A. 2016. Hindered rotor tunneling splittings: an application of the two-dimensional non-separable method to benzyl alcohol and two of its fluorine derivatives. Phys Chem Chem Phys., 18(13), 8945-8953.

[17] Crowder, G. A. 1979. Vibrational Spectra of oFluorobenzyl Alcohol. Journal of Fluorine Chemistry, 14, 77-79.

[18] Liu, C. G., Su, Z. M., Guan, X. H., Muhammad, S. 2011. Redox and Photoisomerization Switching the Second-Order Nonlinear Optical Properties of a Tetrathiafulvalene Derivative Across Six States: A DFT Study. J. Phys. Chem. C, 115, 23946-23954. 